\title{
Steroid resistant hypereosinophilic syndrome found to be Hodgkin's Lymphoma
}

\author{
Christine Joy Licata, Sowmya Arja, Suzanne Teuber \\ Division of Rheumatology, Allergy, and Clinical Immunology, Department of Internal Medicine, UC Davis School of Medicine, \\ Sacramento, CA, USA
}

Received: September 1, 2021

Accepted: October 15, 2021

Online Published: November 16, 2021

DOI: $10.5430 /$ crim.v8n2p10

URL: https://doi.org/10.5430/crim.v8n2p10

\begin{abstract}
Hyperoeosinophilic syndrome (HES) is rare, and clinicians may not recognize its potential association with malignancy. Red flag signs of HES include steroid resistance, older age, and significant lymphadenopathy that can be indicative of malignancy. In this case, an elderly male presenting with right chest wall erythema and axillary lymphadenopathy was initially diagnosed with and treated for cellulitis. Labs were significant for hypereosinophilia. Evidence of end organ damage raised concern for HES. Over the course of three hospitalizations, he was found to have a rising eosinophil count despite high-dose corticosteroid treatment. Further investigation eventually revealed a diagnosis of Hodgkin's Lymphoma. This case highlights steroid-resistant HES as a presenting sign of malignancy and allows for discussion of potential investigative approaches for HES therapy. Though corticosteroids are first-line treatment for hypereosinophilia and HES, they are well known to have many adverse effects. Biologics, such as mepolizumab and benralizumab, have more acceptable side effect profiles and are effective in treating non-myeloid HES. The use of biologics as first-line treatment for HES has yet to be investigated.
\end{abstract}

Key Words: Hypereosinophilic Syndrome, Secondary hypereosinophilia, Red Flag, Hodgkin's Lymphoma, Steroid resistance, Interleukin-5, Biologics

\section{INTRODUCTION}

Hypereosinophilic syndrome (HES) is rare, and the true prevalence is unknown, with one study showing prevalence as low as 0.36 per $100,000 .{ }^{[1]}$ The definition of HES has changed over time. Some have defined it as an absolute eosinophil count (AEC) of greater than $1,500 \mathrm{cells} / \mu \mathrm{l}$ on at least two separate occasions with evidence of persistent end-organ eosinophilic damage. ${ }^{[2]}$ Others simply define it as hypereosinophilia with tissue or organ damage or as hypereosinophilia (HE) present for six months with presumptive tissue damage. ${ }^{[2,3]}$ HES is distinguished from simple HE, which is characterized by AEC greater than 1,500 cells $/ \mu 1$ without end-organ damage. ${ }^{[4]}$ The clinical manifestations of this condition are highly variable, ranging from asymptomatic eosinophilia to end-organ failure ${ }^{[5]}$ Due to its varied presentation, clinicians may not recognize red flag signs of HES and its association with malignancy, which can delay appropriate treatment. In this case, an elderly man was found to have a persistent rise in eosinophil count despite treatment with high-dose corticosteroids. He was diagnosed with Hodgkin's Lymphoma and ultimately died of medical complications. This case highlights red flag signs of HES, the potential for associated malignancy, and allows for discussion of potential investigative approaches for HES therapy.

*Correspondence: Christine Joy Licata; Email: christinelicata19@gmail.com; Address: Division of Rheumatology, Allergy, and Clinical Immunology, Department of Internal Medicine, UC Davis School of Medicine, Sacramento, CA, USA. 


\section{Case presentation}

A 68 year-old male with a history of allergic rhinitis, coronary artery disease status post percutaneous coronary intervention, heart failure with reduced ejection fraction of $35 \%$, hyperlipidemia, hypertension, and depression presented to the emergency department (ED) with right chest wall erythema, chills, fatigue, and dry cough. He was diagnosed with right axillary cellulitis and discharged with a one week course of doxycycline and cephalexin; his white blood cell count was 20.6 cells $/ \mu 1 \times 10^{3}$ with a peripheral AEC of 989 cells $/ \mu 1$ at the time. After initial mild improvement, his symptoms worsened and prompted a return visit to the ED, which ultimately led to hospitalization for further evaluation and treatment. Physical examination was notable for significant axillary lymphadenopathy and persistent right chest wall erythema. Laboratory evaluation was significant for marked leukocytosis, transaminitis, and hypereosinophilia (see Table 1). A chest CT scan showed axillary and rectopectoral adenopathy. He was started on IV vancomycin and piperacillin-tazobactam. Evaluation was negative for unusual infections and helminths (see Table 2), flow cytometry abnormalities and FIP1L1-PDGFRA fusion (see Table 3). Rheumatologic and gastrointestinal evaluations were also negative (see Tables 4 and 5). Lymph node core biopsy showed eosinophilic lymphangitis. After 12 days of hospitalization, he was discharged with cefpodoxime to complete a 14-day antibiotic course. Results of a lymph node excisional biopsy and skin punch biopsy were pending at the time of discharge.

Table 1. Lab trend over hospital course

\begin{tabular}{|c|c|c|c|c|c|}
\hline Week & 1 & 3 & 3 & 4 & 6 \\
\hline $\begin{array}{l}\text { WBC }(\text { cells } / \mu \mathrm{l} \\
\left.\times 10^{3}\right)\end{array}$ & 35.0 & 35.3 & 40.2 & 36.7 & 0.1 \\
\hline $\begin{array}{l}\text { AEC } \\
\text { (cells } / \mu \mathrm{l})\end{array}$ & 1,600 & 5,800 & 9,300 & 11,200 & 0.0 \\
\hline $\begin{array}{l}\text { Trop-I } \\
(\mathrm{ng} / \mathrm{ml})\end{array}$ & - & 0.06 & - & 0.14 & - \\
\hline AST (IU/L) & 116 & 65 & - & 37 & - \\
\hline ALT (IU/L) & 177 & 148 & - & 128 & - \\
\hline ALP (IU/L) & 128 & 172 & - & 332 & - \\
\hline
\end{tabular}

Two days later, he returned to the ED with worsening chest wall erythema, swelling, and fatigue. Physical examination was significant for persistent bulky axillary lymphadenopathy, increased right chest wall erythema and swelling, and a mobilliform rash on the abdomen thought to be drug-induced from vancomycin. Consequently, treatment with IV daptomycin was initiated. Laboratory evaluation revealed persistent leukocytosis, increasing AEC, transaminitis, and elevated troponin-I. Skin punch biopsy performed during his prior hospitalization showed atypical cellular proliferation and dense inflammatory infiltrate with prominent eosinophils. Lymph node excisional biopsy revealed Reed-Sternberg cells within heavy eosinophilic infiltrate consistent with Hodgkin's Lymphoma. Based on these results, prednisone $90 \mathrm{mg}$ daily was started and IV daptomycin was discontinued. Despite this change in therapy, the patient's AEC continued to increase, leading to initiation of hydroxyurea $500 \mathrm{mg}$ twice per day, which was later increased to $1,000 \mathrm{mg}$ twice per day due to lack of response in AEC. He was discharged after eight days on dexamethasone and hydroxyurea with instructions to follow up with hematology/oncology for outpatient treatment of his Hodgkin's Lymphoma.

Table 2. Infectious Disease Evaluation

\begin{tabular}{ll}
\hline Lab Test & Result \\
\hline EBV IgG/IgM & Positive/Negative \\
Hep BcIgM & Negative \\
Hep BsAb & Positive \\
Hep BsAg & Negative \\
HIV & Negative \\
Hep C Ab & Negative \\
Bartonella IgG & Negative \\
Strongyloides & Negative \\
Histoplasma Serum Antigen & Negative \\
Histoplasma Antibody & Negative \\
Histoplasma Urine Antigen & Negative \\
G6PD (u/g) & 726 (high) \\
C Difficile & Negative \\
TB quantiferon & Indeterminate \\
COVID-19 & Negative \\
Blood Culture & No Growth to Date \\
Stool Culture & Normal Fecal Flora \\
Stool Ova \& Parasite & Normal Fecal Flora \\
\hline &
\end{tabular}

Two days later, he again returned to the ED due to extreme fatigue. At this point, his chest wall erythema and swelling had improved, but laboratory evaluation revealed persistent marked leukocytosis, further increase in AEC from his last hospitalization, transaminitis, and elevated troponin. Hydroxyurea was stopped, prednisone was weaned, and he was started on cycle 1 of adriamycin, bleomycin, vinblastine, dacarbazine (ABVD) chemotherapy. After chemotherapy, 
he developed pancytopenia. Unfortunately, he suffered from acute hypoxic respiratory failure from presumed hospitalacquired pneumonia requiring escalation of care to the medical intensive care unit. He was eventually transitioned to comfort care and later died in the hospital.

Table 3. Hematologic/Oncologic Evaluation

\begin{tabular}{ll}
\hline Lab Test & Result \\
\hline Flow Cytometry & Non-specific, no overt increase in blasts \\
Jak2 & Negative \\
BCR-ABL & Negative \\
PDGFRa & Negative \\
PDGFRb & Negative \\
FDFR1 & Negative \\
Tryptase & Normal \\
\hline
\end{tabular}

Table 4. Rheumatologic Evaluation

\begin{tabular}{ll}
\hline Lab Test & Result \\
\hline MPO & Negative \\
IgG subclass $(\mathrm{g} / \mathrm{L})$ & Normal except IgG subclass 3 (137, high) \\
PR3 & Negative \\
ANA & Negative \\
ESR $(\mathrm{mm} / \mathrm{hr})$ & 102 (high) \\
CRP $(\mathrm{mg} / \mathrm{L})$ & $>200$ (high) \\
CK $(\mathrm{U} / \mathrm{L})$ & $<10$ (normal) \\
Myoglobin & Normal \\
Aldolase $(\mathrm{u} / \mathrm{dL})$ & 11.6 (high) \\
\hline
\end{tabular}

Table 5. Gastrointestinal Evaluation

\begin{tabular}{ll}
\hline Lab Test & Result \\
\hline ASMA & Negative \\
Antimitochondrial Antibody & Negative \\
IgG $(\mathrm{g} / \mathrm{L})$ & 832 (normal) \\
CMV PCR qualitative & Positive \\
HSV PCR & Negative \\
Ceruloplasmin (mg/dL) & 37.1 (high) \\
Alpha 1 anti-trypsin $(\mathrm{mg} / \mathrm{dL})$ & 340 (high) \\
INR & 1.79 \\
\hline
\end{tabular}

Table 1 shows the trend in certain lab values corresponding to weeks following initial admission. Following initiation of antibiotics on week 1 and high-dose corticosteroids on week 3, there was no effect on white blood cell count (WBC) or absolute eosinophil count (AEC), and AEC continued to increase. Troponin-I and liver function tests demonstrated eosinophilic end organ damage. Data from week 6 reflects pancytopenia following ABVD chemotherapy administration.

\section{Discussion}

Delay in the recognition, evaluation, and treatment of HES and failure to recognize red flag signs can be detrimental. This case illustrates an example of secondary HES from
Hodgkin's Lymphoma initially believed to be primary HES due to persistently elevated troponin and transaminitis indicative of heart and liver damage. The most common clinical manifestation of HES is dermatologic in nature and may manifest as a rash, angioedema, or urticaria. ${ }^{[6]}$ In fact, there is estimation that $50 \%$ of patients with HES have pruritic erythematous macules, papules, plaques, wheals, nodules or other skin lesions during the course of the disease, particularly on the hands or flexural areas. ${ }^{[7,8]}$ One of the defining clinical features in this case was persistent right chest wall erythema that had been mistakenly diagnosed as cellulitis. There are a number of ways an HES rash can present, including that depicted in Figure 1. The second most common manifestation is lung fibrosis from eosinophilic infiltration causing cough and shortness of breath. Other organ systems commonly affected in HES include gastrointestinal (eosinophilic gastritis), cardiac (eosinophilic myocarditis), and neurologic (encephalopathy, sinus thrombi, cerebral thromboemboli). ${ }^{[6]}$

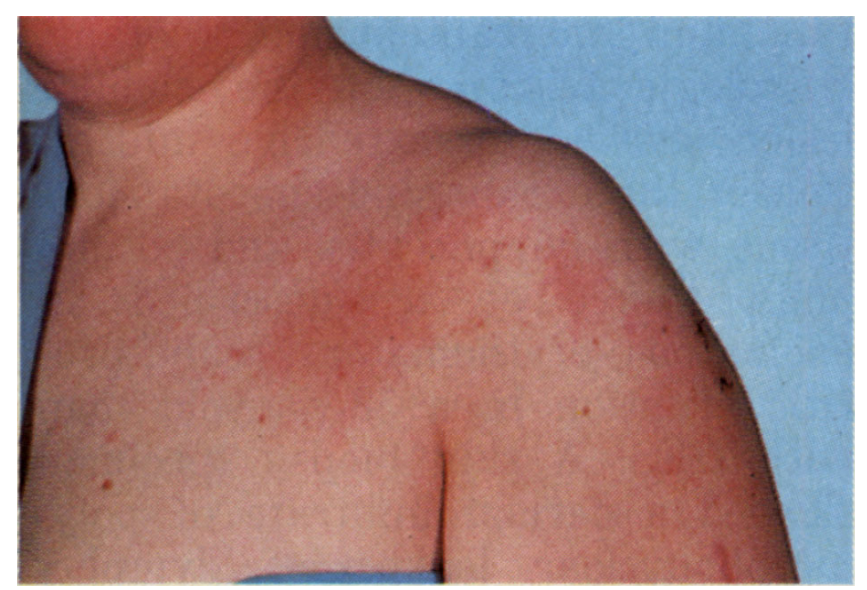

Figure 1. Example of Dermatologic Manifestation of HES

The example above is taken from Kazmierowski et al. ${ }^{[15]}$ and demonstrates an example of an erythematous papular rash that can be associated with HES. Photographic documentation of the rash discussed in this case was not available for review, but this example may be similar in appearance.

HES is classified as primary, secondary, or idiopathic according to the underlying pathogenesis. There are certain hematological malignancies associated with secondary HES. In a retrospective study by Mayo Clinic, non-Hodgkin's lymphoma was the most common of malignancies found to be associated with HES, followed by chronic lymphocytic leukemia and eosinophilic leukemia. ${ }^{[9]}$ It is thought that that the relationship between HES and Hodgkin's Lymphoma is linked to the abnormal proliferation of T cells in Hodgkin's Lymphoma, which leads to overproduction of eosinophils 
that then deposit in tissue. ${ }^{[10]}$

There are certain variants of HES associated with gene defects in the tyrosine kinase receptor platelet-derived growth factor (PDGFR) and lymphocytic variants. As such, an important part of the oncologic evaluation of HES involves looking for a mutation in Fip1-like1-platelet-derived growth factor receptor alpha gene (FIP1L1-PDGFRA), which results in clonal eosinophilic production and is associated with the myeloid variant of HES. ${ }^{[11]}$ In the initial stages of clinical evaluation, if AEC is greater than 1,500 cells/ $\mu$ l, end-organ damage should be assessed immediately. The patient in this case was found to have transaminitis and elevated troponin-I suggestive of eosinophilic deposition. Infectious etiologies, particularly parasitic infection, must be investigated when initiating high dose corticosteroid treatment, in order to avoid a fatal Strongyloides hyperinfection.

Initial treatment of HES includes high-dose glucocorticoids with the goal of reducing AEC below 1,500 cells/ $\mu$ l to prevent or avoid further end organ damage. ${ }^{[12]}$ Those with myeloid-variant HES may be started on imatinib, a tyrosine kinase inhibitor. The expected response to high-dose glucocorticoids is a dramatic drop in AEC by more than $50 \%$ of the original value within 24 hours or at least stability in the value. ${ }^{[13]}$ Steroid resistance and other red flags, including significant lymphadenopathy, older age onset, and end-organ damage should raise concern for underlying malignancy, as demonstrated in this patient's case.

For primary or idiopathic HES recalcitrant to corticosteroids, treatment options include biologic therapies that target IL-
5 or its receptor, such as mepolizumab and benralizumab respectively. Interleukin-5 (IL-5) is an important hematopoietic cytokine that is specific for eosinophil differentiation. ${ }^{[14]}$ Though high-dose corticosteroids are accepted as first line initial therapy, their use is often limited by myriad adverse effects. In contrast, mepolizumab and benralizumab have more favorable side effect profiles and may eventually be considered as initial therapy for non-myeloid HES.

Figure 1 is the example which is taken from Kazmierowski et $\mathrm{al}^{[15]}$ and demonstrates an example of an erythematous papular rash that can be associated with HES. Photographic documentation of the rash discussed in this case was not available for review, but this example may be similar in appearance.

\section{Conclusion}

Overall, HES is rare but can be fatal if not recognized appropriately. HES can be primary, secondary, or idiopathic in etiology. In this case, the patient was eventually diagnosed with secondary HES from Hodgkin's Lymphoma following recurrent ED visits and hospitalization and failed treatment for infection and primary HES. It is important to recognize red flag signs, signs of end-organ damage, and other evidence of malignancy. Initial treatment of HES typically involves high-dose corticosteroids. The use of biologics targeting IL5 and its receptor as initial treatment for non-myeloid HES remains to be investigated.

\section{Conflicts of InTEREST Disclosure}

The authors have declared no conflicts of interest.

\section{REFERENCES}

[1] Crane MM, et al. Incidence of myeloproliferative hypereosinophilic syndrome in the United States and an estimate of all hypereosinophilic syndrome incidence. The Journal of allergy and clinical immunology, 2010;126:179. PMid:20639012. https://doi.org/ $10.1016 / j \cdot j a c i .2010 .03 .035$

[2] Simon HU, et al. Refining the definition of hypereosinophilic syndrome. Journal of Allergy and Clinical Immunology. 2010; 126: 45-49. PMid:20639008. https://doi.org/10.1016/j.jaci. 2 010.03 .042

[3] Stella S, et al., Molecular Pathogenesis and Treatment Perspectives for Hypereosinophilia and Hypereosinophilic Syndromes. International Journal of Molecular Sciences. 2021; 22: 486. PMid:33418988. https://doi.org/10.3390/ijms22020486

[4] Valent P, et al. Contemporary consensus proposal on criteria and classification of eosinophilic disorders and related syndromes. Journal of Allergy and Clinical Immunology. 2012; 130: 607-612. PMid:22460074. https://doi.org/10.1016/j.jaci. 2012.02 .019

[5] Curtis C, Ogbogu P. Hypereosinophilic syndrome. Clinical Reviews in Allergy \& Immunology. 2016; 50: 240-251. PMid:26475367. https://doi .org/10.1007/s12016-015-8506-7

[6] Ogbogu PU, et al. Hypereosinophilic syndrome: a multicenter, retrospective analysis of clinical characteristics and response to therapy. Journal of Allergy and Clinical Immunology. 2009; 124: 1319-1325. PMid:19910029. https://doi.org/10.1016/j.jaci.2009.09 .022

[7] Leiferman KM, Gleich GJ, Peters MS. Dermatologic manifestations of the hypereosinophilic syndromes. Immunology and allergy clinics of North America, 2007; 27: 415-441. PMid:17868857. https://doi.org/10.1016/j.iac.2007.07.009

[8] Williams KW, et al. Hypereosinophilia in children and adults: a retrospective comparison. The Journal of Allergy and Clinical Immunology: In Practice. 2016; 4: 941-947. PMid:27130711. https: //doi.org/10.1016/j.jaip. 2016.03.020

[9] Jin JJ, Butterfield JH, Weiler CR. Hematologic Malignancies Identified in Patients with Hypereosinophilia and Hypereosinophilic Syndromes. J Allergy Clin Immunol Pract. 2015; 3: 920-925. PMid:26342744. https://doi.org/10.1016/j . jaip.2015.06 .009

[10] Blomme E, et al. Hypereosinophilic syndrome with Hodgkin's-like lymphoma in a ferret. Journal of Comparative Pathology. 1999; 120: 
211-217. PMid:10087494. https://doi.org/10.1053/jcpa.1 998.0203

[11] Montgomery ND, et al. Diagnostic complexities of eosinophilia. Archives of pathology \& laboratory medicine. 2013; 137: 259-269. PMid:23368869. https://doi.org/10.5858/arpa.2011-059 7-RA

[12] Kuang FL, Klion AD. Biologic agents for the treatment of hypereosinophilic syndromes. The Journal of Allergy and Clinical Immunology: In Practice. 2017; 5: 1502-1509. PMid:29122152. https://doi.org/10.1016/j.jaip.2017.08.001

[13] Khoury P, et al. Hypereosinophilic syndrome subtype predicts re- sponsiveness to glucocorticoids. The Journal of Allergy and Clinical Immunology: In Practice, 2018; 6: 190-195. PMid:28757367. https://doi.org/10.1016/j.jaip.2017.06.006

[14] Di Biagio E, et al. Eosinophilia in Hodgkin's disease: a role for interleukin 5. International Archives of Allergy and Immunology. 1996; 110: 244-251. PMid:8688671. https://doi.org/10.1159/0002 37294

[15] Kazmierowski JA, et al. Dermatologic manifestations of the hypereosinophilic syndrome. Archives of Dermatology. 1978; 114: 531-535. PMid:646363. https://doi.org/10.1001/archderm.1978.01 640160009003 